\title{
Instantiations of an ERP Implementation Methodology: A Critical Realist Case Study
}

\author{
Daniela Mihailescu \\ Uppsala University, Campus \\ Gotland, Sweden \\ daniela.mihailescu@im.uu.se
}

\author{
Marius Mihailescu \\ Uppsala University, Campus \\ Gotland, Sweden \\ marius.mihailescu@im.uu.se
}

\begin{abstract}
New or revised implementation methodologies are introduced by Enterprise Resource Planning (ERP) systems vendors to improve the results of ERP systems implementations. In spite of an implementation methodology's potential and significance for practice, little attention has been paid to this object of study in enterprise systems literature. Moreover, current studies reveal contradictory findings and provide a fragmented understanding. The purpose of this paper is to describe and explain the emergence of an implementation methodology instantiations through the lens of a realist social theory underpinned by critical realism. Our qualitative study provides explanatory insights and a rich description of an implementation methodology instantiations generated over time. This study also provides guidance for practitioners, to anticipate potential implementation methodology instantiations and identify the conditions contributing to their occurrence and change, and contribute to a more coherent view on implementation methodology instantiations as an issue of organisational change.
\end{abstract}

\section{Introduction}

Information systems development (ISD) in organizations was traditionally achieved through an inhouse approach which involves the development of IS to address the particular information needs of an organization. However, by early 2000 with the advent of Enterprise Resource Planning (ERP) systems, which are organizational-wide systems based on integrated generic application packages [19], ISD was accomplished through a buy-in package approach. The objectives and principles of this alternative approach, e.g. business process re-engineering and rapid implementation based on reference models, are incorporated in computer-based implementation methodologies provided by ERP vendors and their implementation partners. For instance, SAP AG recommends the "AcceleratedSAP" (ASAP) methodology for implementing SAP solutions and Microsoft recommends the "Sure Step" methodology for implementing Microsoft Dynamics solutions. Iivari and Huisman [13] define Implementation Methodology (IM) as a collection of interrelated components, such as methods, techniques, toolsets and services underpinned by a buy-in package approach intended to provide production, control, cognitive, cooperation, and infrastructural support [13]. IM is intended to support an implementation process and emphasize: a mix of business process design and change [7]; configuration or customization of application packages [12]; and project management and evaluation [17].

Although the potential value of IM is disputed [24], the use of IM is still an intriguing case which deserves further examination because: a) unlike Information Systems Development Methodology (ISDM), which focus on the development of isolated and functionbased software, IM emphasises organisational aspects; b) time and budgets overruns have been more a rule than an exception, and the value of IM for implementation of ERP, has been questioned [2]; and c) IM, which is a type of complex innovation technology; it imposes a substantial knowledge burden on adopters and might reduce their performance or impede its use [11].

Despite their different focus, i.e., ISDM in development of IS, and IM in implementation of ERP, both share the same objective in improving the process and the product delivered to customers. Consequently, the available ISD research provides an appropriate starting point to explore the potential characteristics of this type of objects. Based on different assumptions and theoretical schools, two literature streams dominate ISD research: a method engineering stream, which focuses on ISDM and its development, and a socio-organizational stream, which focuses on the use of ISDM and its adaptation [26]. The two streams are dominated by either a positivist perspective, dominating on method engineering stream, or an interpretive perspective, dominating on socioorganizational stream. 
These perspectives fail to provide causal explanation for changes that emerge over time in IM instantiations [23]. Critical realism perspective promises to resolve those problems. There is growing interest in the application of critical realism and particularly the morphogenetic approach [3] within IS research. Scholars [16] illustrate the benefits of critical realism when exploring the IS artefact, highlighting its efficiency for analyzing complex contexts. However, to date, there are few empirical studies where the morphogenetic approach has been applied in organizational settings [27]. What makes critical realism and the morphogenetic approach of particular relevance for this study is their focus on providing explanations of change over time.

The objective of this study is to explain the emergence of an IM through the lens of Realist Social Theory's morphogenetic approach underpinned by critical realism. More specifically we address the following RQ: How are implementation methodology instantiations generated over time? The research question is addressed through an intensive, longitudinal case study of one IM, which is included on the service portfolio of SAP AG, who takes an active role in the implementation of their products. SAP AG's implementation methodology, ASAP, is recommended as a de facto standard and is made available free of charge by the vendor for all implementation projects. In addition, a strengthened connectivity between product and services and involvement of the vendor in implementation projects take place.

The knowledge developed in this study has an explanatory character and the efforts are directed toward opening the 'black box' of changes in adopting IMs in ERP implementations. The description and potential explanation is based on ISD research and is enhanced by empirical findings. Consequently, an extended and systematic way to organize IM consideration and its instantiations is provided. Besides the theoretical contribution, the results can help ERP professionals, i.e. practitioners as well as academics, to become aware of the characteristics of IM and its instantiations. This study also responds to the request for a more explicit consideration of critical realism in general, and morphogenetic approach in particular, in IS research [9].

The rest of the study is structured as follows: prior research on IS development methodology is briefly presented in Section 2 . Section 3 introduces the theoretical basis of the research and provides a brief description of critical realism and the morphogenetic approach [1]. Then, the research design and findings are presented, followed by a discussion of the implications of the findings. The study concludes with limitations and future research.

\section{Related literature}

Implementation Methodology is one of the latest approaches in ISDM initiatives. Since this type of initiative originated in the late 1960s [4], there is a large and heterogeneous body of ISDM research. The review presented in this section highlights four theoretical perspectives that have been applied in the interpretation of ISDM.

A system perspective - ISDM is interpreted as a collection of interrelated components such as: paradigm, approach, method, technique [12], development tools, and services [4].

A structural perspective - ISDM is interpreted from a structuration perspective, which provides the view of ISDM as a socially constructed means, with enabling or constraining potential [20].

A knowledge and learning perspective - ISDM is regarded as an object that can be transferred through some form of communication from a supplier side [5], and assimilated through learning on the adopter side [11]. ISDM has also been interpreted from a learning perspective [21]. Yet, ISDM is not a holder of knowledge but an evolving artifact which becomes understandable and meaningful as it is used.

An innovation perspective - The characteristics of ISDM perceived by systems developers to influence deployment are: relative advantage; usefulness; compatibility; and trialability $[22 ; 12]$. The two constructs, relative advantage and usefulness, are similar and highlight the individual's performance expectancy [25]. The other two constructs compatibility and trialability are considered significant to facilitate intention, formation, and use. An emphasis on the individual developer's perceptions is considered too narrow to be of much use for organizations which instantiate ISDM, because it underemphasizes the challenges and the role of adopters [18]. Therefore, researchers drawing on a knowledge diffusion perspective have focused their attention on analyzing the barriers that can impede the transfer or the integration of knowledge within or across organizations and communities.

Each perspective provides a different view on ISDM and its potential roles in different contexts. The literature review discloses the complex and ambiguous nature of ISDM and its potential not only to support but also to induce changes and improvements. Hence, two particular issues emerge: 1) divergent findings and fragmented understanding of ISDM, which, as we see it, might be related to a narrow interpretation; and 2) a shift of focus from contents and potential individual improvements in performance, towards an emphasis on potentials and challenges in the development and deployment of ISDM at a collective level. 


\section{Theoretical Foundation}

To address the research question, we applied Archer's morphogenetic approach [1;3], which falls under the critical realism umbrella. Ontologically, critical realism distinguishes between three ontological domains: the empirical domain, which consists of what we experience; the actual domain, in which events occur if the mechanisms are activated; and the real domain, which consists of underlying structures, and mechanisms. The generative mechanisms residing in the real domain exist independently of the patterns of events that they are capable of generating. Epistemologically, critical realism focuses on the relationship between mechanisms, events, and our empirical experiences [15].

In order to explain how IM instantiations emerge over time, the morphogenetic approach $[1 ; 3]$ is used. The morphogenetic approach reflects critical realism's assumptions and is probably the most commonly applied in critical realism studies [8]. By artificially separating the dialectical interplay between structure and agency, the morphogenetic approach helps us to study how structure shapes action and social interaction (i.e., agency), and how agency changes (i.e., morphogenesis) or reproduces (i.e., morphostatis) a given structure. The analytical structure-agency dualism is operationalized by means of a morphogenetic cycle that consists of three phases: structural conditioning; social interaction; and structural elaboration [3], (figure 1).

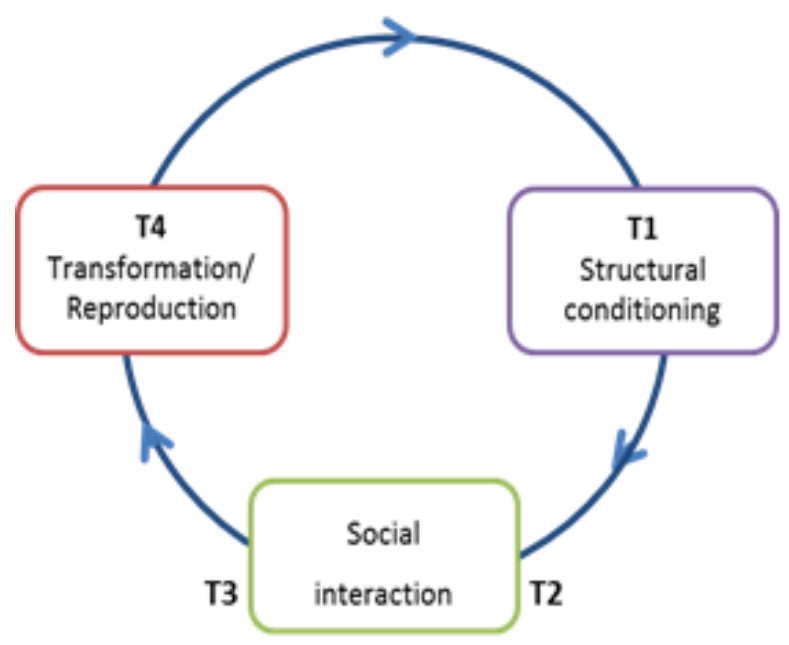

Figure 1. The morphogenetic cycle

\section{A realist conceptual framework}

The morphogenetic approach provides both an explanatory framework for examining interactions between structure and agency, and a theoretical lens for explaining the emergence of IM over time. Our conceptualization makes possible to describe and explain IM instantiations as a process of change generated by interplay between structural conditioning and agential interaction over time.

The morphogenetic analysis starts at T1, structural conditioning, and represents the start of a morphogenetic cycle. The morphogenetic cycle is the result of the actions of previous agents. The interplay between the properties of the socio-cultural structures and the IM give rise to four situational logics [1]:

Compromise, i.e., when relationships between elements of a structure are necessary but contradictory with each other, agents compromise in order to maintain their structure;

Protection, i.e., when the relationships between the elements of a structure are necessary and complementary, agents are motivated to protect the existing structure;

Elimination, i.e., when the relationships between the elements of a structure are contingent but contradictory, agents are motivated to eliminate the contradiction to maintain the status quo; and

Opportunity, i.e., when the relationships between the elements of a structure are contingent and complementary, agents exploit the new opportunities in order to increase their capabilities. Situational logics provide "directional guidance" [1] for different forms of strategic action and motivate agents to maintain or alter the status quo due to different systemic relationship. If structures are:

Necessary but contradictory, then ERP implementers compromise in order to keep the structures;

Necessary and complementary, then ERP implementers protect the existing structures;

Contingent but contradictory then ERP implementers eliminate the incompatibility; and

Contingent and complementary, then ERP implementers take advantage of new opportunities (Figure 2). 


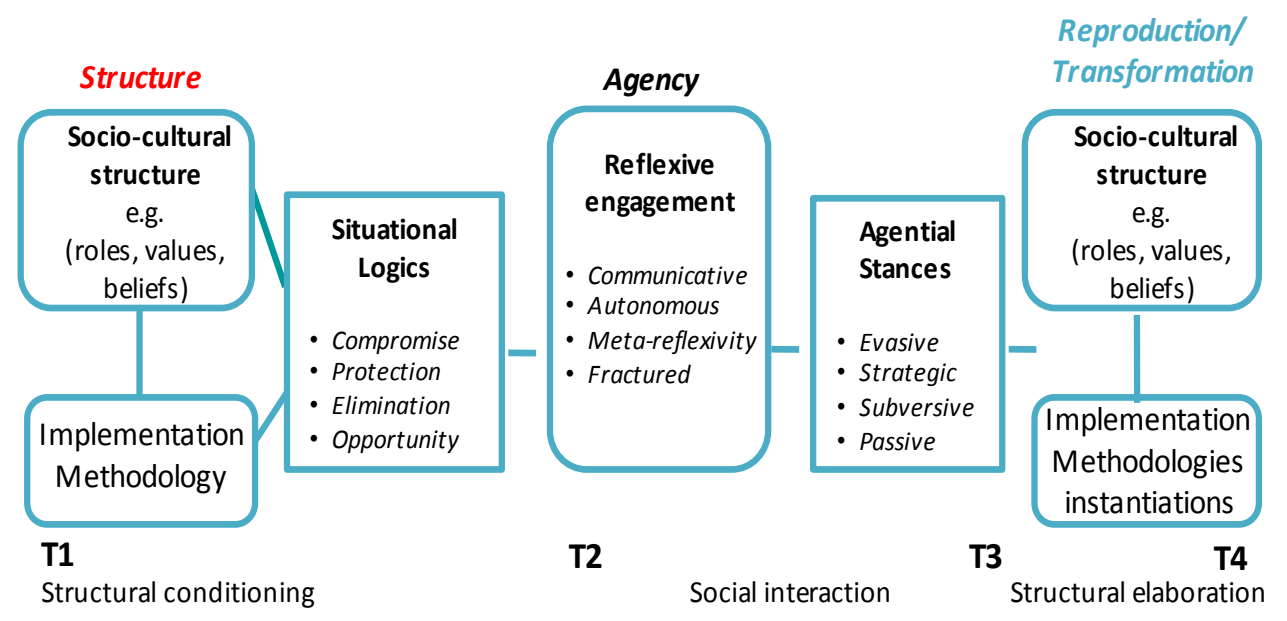

Figure 2. A realist conceptualization of IM instantiation

Social interaction, at T2-T3, corresponds to the interaction of the agency, which reflexively decides on a course of action given their situational logics, and takes a stance towards their context. In other words, EPR implementers' stances express their ultimate concerns and inform their actions and interactions in an EPR implementation context. The potential stances, related reflexive properties, and ultimate concerns [2], are illustrated in table 1.

Table 1. Implementer's stances, reflexive properties and ultimate concerns

\begin{tabular}{|l|l|l|}
\hline \multicolumn{1}{|c|}{ Stances } & \multicolumn{1}{|c|}{$\begin{array}{c}\text { Reflexive } \\
\text { property }\end{array}$} & \multicolumn{1}{|c|}{$\begin{array}{c}\text { Ultimate } \\
\text { concerns }\end{array}$} \\
\hline Evasive & Communicative & $\begin{array}{l}\text { Inter-personal } \\
\text { relationships }\end{array}$ \\
\hline Strategic & Autonomous & $\begin{array}{l}\text { Performative } \\
\text { achievements }\end{array}$ \\
\hline Subversive & Meta & $\begin{array}{l}\text { Value } \\
\text { rationality }\end{array}$ \\
\hline Passive & Fractured & Disconcerted \\
\hline
\end{tabular}

The first three stances indicate an active agent with a distinctive stance towards his or her situation. The last stance indicates a passive agent who lacks a stance towards his or her environment. The different stances have consequences for the IM instantiations.

Structural elaboration, at T4, represents the effects of the social interactions from the previous phases in the form of structural elaboration, i.e., reproduction (morphostatis) or transformation (morphogenesis). Based on this view, we conceptualize IM instantiation as an emergent process generated by the interplay between: (i) situational logics, which are characterized by socio-cultural configurations that provide directional guidance by enabling or constraining different forms of strategic action; and (ii) agents' stances, which are characterized by self-determined configurations of concerns outlined by different modes of reflexivity motivate agents to pursue different actions and interactions. The interplay between structure (i), and agency (ii), explains the emergence of IM and its instantiations over time, as a morphogenetic process. The results form the start and structural conditioning of the next morphogenetic cycle at $\mathrm{T} 1$.

\section{Research approach}

The research design involves an iterative research process and is based on a longitudinal case study and a qualitative approach for data collection and analysis. The case selected for analysis is represented by SAP's AcceleratedSAP (ASAP) methodology as one methodology instance that is well documented in research publications [10] is used. The case also facilitates familiarization with implementation methodology practice [6]. Our longitudinal study aims to establish a link between Realist Social Theory's morphogenetic approach, and empirical account, i.e., ASAP case. The morphogenetic approach provides a realist framework which is used to describe and explain the instantiations of ASAP implementation methodology in an ERP-implementation context.

The study adopts a qualitative multi-method approach, which subsumes a combination of different qualitative data collection techniques. Data on ISDM and its use is collected through qualitative literature review, while data on ASAP and its use is gathered through interviews and documents.

Due to the focus of this study on the use of IM by implementers, the AIS basket-of-IS journals were included in our search. Google Scholar was used to search through web databases such as ABI/Inform, SpringerLink, and ACM Digital Library. Based on 
insights from the literature review a realist conceptualization of IM instantiation was developed (Figure 2). To gain the insights necessary to explain ASAP's IM as it unfolded over time, we relied on interviews with implementers and ASAP documents.

For the ASAP case, two qualitative data-collection techniques are employed in order to gather evidence from interviews and secondary data. The secondary data consists of documents provided by interviewees as well as additional documents collected from SAP's web place, SAP Conferences and published articles with focus on ASAP. While customers of SAP vendor and its partners could accept and experience the use of ASAP or Powered by SAP methodologies, it was the implementers who provided and possessed not only experiences but also knowledge about these methodologies and used them in ERP implementation projects. Primary data gathered through semistructured interviews consists of 21 interviews with implementers from SAP vendor and its implementation partners (table 2).

Table 2. Summary of Interviewees

\begin{tabular}{|l|l|l|}
\hline \multicolumn{1}{|c|}{ Organization } & \multicolumn{1}{|c|}{ Position } & Time \\
\hline SAP Sweden & Senior consultant & 2h \\
\hline $\begin{array}{l}\text { Bore Dahlberg } \\
\text { Consulting Sweden }\end{array}$ & Senior consultant & $2 \mathrm{~h}$ \\
\hline $\begin{array}{l}\text { Spring Consulting } \\
\text { Sweden }\end{array}$ & Senior consultant & $1 \mathrm{~h}$ \\
\hline $\begin{array}{l}\text { Spring Consulting } \\
\text { Sweden }\end{array}$ & Management consultant & $1 \mathrm{~h}$ \\
\hline SAP Sweden & Senior business consultant & $2 \mathrm{~h}$ \\
\hline SAP America & $\begin{array}{l}\text { SAP Solution Manager } \\
\text { consultant }\end{array}$ & $45 \mathrm{~m}$ \\
\hline Cap Gemini US & Senior consultant & $45 \mathrm{~m}$ \\
\hline $\begin{array}{l}\text { Chrysalis Consulting } \\
\text { Services US }\end{array}$ & Senior consultant & $45 \mathrm{~m}$ \\
\hline $\begin{array}{l}\text { IBM Business } \\
\text { Consulting Services US }\end{array}$ & Senior consultant & $45 \mathrm{~m}$ \\
\hline Deloitte Consulting US & Consultant & $30 \mathrm{~m}$ \\
\hline $\begin{array}{l}\text { Spring Consulting } \\
\text { Sweden }\end{array}$ & Senior consultant & $2 \mathrm{~h}$ \\
\hline IBM Consulting US & Senior consultant & $1 \mathrm{~h}$ \\
\hline SAP Sweden & Senior business consultant & $1 \mathrm{~h}$ \\
\hline SAP Denmark & Senior consultant & $1 \mathrm{~h}$ \\
\hline SAP & $\begin{array}{l}\text { SAP Solution Manager } \\
\text { consultant }\end{array}$ & $45 \mathrm{~m}$ \\
\hline CIBER Sweden & Senior consultant & $1 \mathrm{~h}$ \\
\hline SAP Sweden & Senior support adviser & $1 \mathrm{~h}$ \\
\hline IBM Consulting Sweden & Senior consultant & $1 \mathrm{~h}$ \\
\hline SAP Sweden & Senior consultant & $1 \mathrm{~h}$ \\
\hline Cap Gemini Sweden & Senior consultant & $45 \mathrm{~m}$ \\
\hline Accenture Sweden & Senior consultant & $45 \mathrm{~m}$ \\
\hline
\end{tabular}

Data from the interviews was gathered at three times and serves different purposes. The first ten interviews provide information about complementary aspects and interrelated components of ASAP and its use in ERP implementation context. All participants from the first round of interviews are contacted for follow-up interviews. Only three of them participated in the second round of interviews, helping in focusing the orientation of the study and seconding the insights about the significance of adaptation and interaction from the first stage of the research. The last eight interviews provide additional insights into relationships among interrelated components of ASAP adopted by ERP implementers.

The data analysis starts with a retrospective analysis of IM instantiation in the interviews and document analyses, evidence for content and purpose is determined by the presence or lack of presence of approach, process, activities, tools, services, training, coordination or organisational support.

Next step in the analysis assess the IM instantiations, and hence the focus shifts towards two dimensions, i.e., situational logics and implementers' stance. The first dimension, which is represented by situational logics, is examined by the shape of distribution of material resources, like wealth, power or expertise, and ideational resources, like theories or beliefs, as structural-cultural configurations. These configurations result in structural relationships between roles and activities on SAP implementation, and ideational relationships between ASAP, implementation process and SAP product.

The shape of resource distributions regarding financials, expertise, ideas or beliefs contributing to complementarities or contradictions and necessary or contingent relationships among structural and cultural components, serve as indicators for particular situational logics.

The second dimension, which is represented by agents' (implementers) stances, is considered according to implementers' ultimate concerns, which characterize dominant modes of reflexivity. Lack of, or predominance of concerns for productivity, quality of products or relationships characterising different modes of reflexivity serve as indicators for particular stances.

Our causal analysis reveals four morphogenetic cycles in the emergence of IM instantiations over time. As a result of iterations between different cycles both in the first and second step in the analysis, multidimensional changes and different IM instantiations are identified. 


\section{Findings}

We employ the suggested framework and describe the four morphogenetic cycles that explain the different IM instantiations generated over time. The examination follows the structural conditioning, social interaction (situational logics and agents' stances) and structural elaboration phases of the morphogenetic cycle which are described below.

The first cycle indicates the occurrence of a fragmented instantiation and covers the time span between early1980s and 1989.

T1 - Structural conditioning: available on the market as early as the 1970s, generic application packages began to attract increased interest among customers in the early 1980s. One of the few suppliers of generic software applications and contributors to the growth of the software industry was SAP AG. By the early 1980s, the company develops and provides a configurable and mainframe-based business application with integrated modules, known as R/2.

The underlying ideas of providing a configurable/customizable product are to reduce potential problems related to the development cycle of the application package and to facilitate a rapid implementation. By the early 1980s, these ideas become rather different and challenge the prevailing in-house IS development approach and the view of using technology to support and automate an organization's existing functions.

The relationship of necessary but contradictory ideas generates a situational logic of correction.

T2-T3 - Social interaction - guided by a situational logic of correction, but dominated by a mode of fractured reflexivity, which is characterized by undetermined concerns and disconcerted experiences, SAP implementers take a passive stance in their orientation towards SAP implementation context. They attempted to advance the alternative principles of a buy-in approach, but also sought to reuse their expertise in IS development. The difficulties encountered in articulating and prioritizing their concerns about $\mathrm{R} / 2$ implementation confined implementers as a dependent and subordinated collectivity controlled by the $\mathrm{R} / 2$ developers.

A limited extension of the content of IM, by adding cognitive and production support is realized.

T4 - Structural elaboration: the effects of passive implementers in a corrective situation contributed to a fragmented instantiation of IM. Passive implementers serve the interests of the $\mathrm{R} / 2$ developers who promote a buy-in package approach, as an alternative to in-house development in a centralized organization.
The second cycle indicates the occurrence of an aggregated instantiation and covers the time span between early 1990s and 1993.

T1 - Structural conditioning: by early 1990s SAP introduces a new generic application package called $R / 3$. The generic application package is based on a client-server architecture, which provides the benefits of portability, inter-operability, and scalability. To benefit from the business processes embedded in the application package, the customer is required to reengineer its business processes in accordance with the $\mathrm{R} / 3$ product. Both $\mathrm{SAP}$ and its partners retain their operational autonomy and differentiate themselves by specializing in complementary areas of expertise. In the case of SAP America, these are exclusively managed by its independent partners on integration, project management, and customer services. Part of SAP and its partners' protective efforts entails reusing the available but limited content of the SAP implementation methodology and reproducing their own expertise. Informed by the principle of the buy-in approach with configuration of generic application packages and project management, the content of the implementation methodology is extended as a complementary part of the implementation process of the SAP generic application packages. The systematization of ideas fostered by SAP and its allies coincides with a protective effort to preserve their own operations and differentiated roles in the development and implementation of generic application packages. This relationship of necessary and complementary roles and ideas generates a situational logic of protection.

T2-T3 - Social interaction - guided by a situational logic of protection and dominated by a mode of communicative reflexivity, which is characterized by an ultimate concern for maintaining concordant interrelationships, SAP and its partners were evasive in their orientation towards the SAP implementation context. They conceived their operations within available but differentiated resources. Their interaction, realized through cooperation, was based on shared interests and an active but circumventing response intended to avoid potential constraints due to a lack of, or incongruent expertise in different SAP implementation areas. Contentment with their position insulated against external stimuli and sustained the reproduction and efficiency of their own operations and expertise.

A horizontal expansion of the implementation method content with added control and analysis support provided by independent partners is realized.

T4 - Structural elaboration: the effects of evasive implementers in a protective situation contributed to an aggregated instantiation of IM. Evasive partners with 
shared interests in their interrelationship are promoting a systematization of a buy-in package approach, in a sectional organization of independent roles and differentiated distribution or resources.

A reduced variety among implementation partners emerged as unintended side-effects in SAP implementation context.

The third cycle indicates the occurrence of an integrated instantiation and covers the time span between 1993 and 1995 .

T1 - Structural conditioning: after the release of the $\mathrm{R} / 3$ application, the expertise necessary to implement it was shallow. In addition, partners used their own IM, which often were better suited to system development than to the task of implementing R/3. SAP America's lack of commitment in the implementation process and the complexity of the $R / 3$ are issues of discontentment and concern for customers who are increasingly dissatisfied with the partners' performance, support and knowledge about $\mathrm{R} / 3$.

In order to tackle this challenge, a team of experienced consultants having participated in joint SAP implementations was assigned to develop an implementation method. Guided by their own sectional interests, they pursued the opportunity to differentiate their operations and services. The team extends the underlying ideas and implementation process and exposes a complementary IM with congruent content.

The relationship of contingent and complementary roles and resources accompanied by necessary and complementary ideas sponsored by SAP and legitimized by an increasing number of customers generates a situational logic of opportunity.

T2-T3 - Social interaction - guided by an opportunistic situational logic and dominated by a mode of meta-reflexivity, which is characterized by concerns about exposing best practices, implementation partners were subversive in their orientation towards the SAP implementation context. They concentrated on providing a specialized set of ideas and resources that were compatible with a SAP implementation role. Their interaction achieved through collaboration was based on a commitment to integrate resources in an attempt to improve quality and raise value rationality in SAP implementation.

A vertical extension of the IM content with added cooperation and representation support provided by an implementation team is realized.

T4 - Structural elaboration: The effects of subversive implementers in opportunistic situation contributed to an integrated instantiation of IM. A subversive team of collaborative partners with shared interests in value rationality and quality was stimulating a systematization of an implementation method in a cohesive organization of interrelated roles and similar distribution of resources.

A display of the alternative values that might animate the interests of passive and diversified partners has emerged as an unintended side-effect.

The fourth cycle indicates the occurrence of an infrastructural instantiation between early 1996 -

T1 - Structural conditioning: by 1996, the outcome of the initiative taken by SAP America and the work of the implementation team was represented by an IM that had been added to SAPs product development portfolio and extended their complementary services. The IM was introduced to SAP's partners and customers as ASAP, and was recommended as a de facto standard for all SAP implementations. In 1999, ASAP was enhanced via implementation of related support tools such as the SAP Solution Manager platform, which was integrated into all SAP installations free of charge and was thereafter owned by the customer. The platform came to be part of SAP's NetWeaver platform. Some of SAP's implementation partners initiated the development and deployment of their own methodologies by extending and/or integrating elements of ASAP. Others deployed ASAP and collaborated with the vendor to enhance it. Positive results regarding efficiency and effectiveness were indicated by SAP and its service partners after the introduction of ASAP. In addition, SAP took a more active role in supporting customers' implementation projects and required the involvement of a SAP representative in all projects. The initiative was met with mixed feelings.

The relationship of contingent and complementary roles and resources accompanied by contingent and complementary ideas legitimized by implementation partners and an increasing number of customers generates a situational logic of opportunity.

T2-T3 - Social interaction: guided by a situational logic of opportunity and dominated by an autonomous mode of reflexivity, which is characterized by an ultimate concern on proficiency and feasible performance achievements, SAP took a strategic stance towards its implementation context. Aware of the limitations and benefits of SAP implementation, a dedicated SAP team harnessed the compliance of various resources and circumvented certain constraints through an active and coordinated interaction.

A connective extension of the IM content with organizational, coordination and production support in a comprehensive platform of service and resources is realized.

T4 - Structural elaboration: The effects of strategic implementers in opportunistic situation contributed to an infrastructural instantiation of IM. A strategic SAP team with particular interests in task and productivity 
achievements was promoting a specialization of distinctive roles and diversified distribution of resources. Intensification of sectional and divergent interests and reduction of interaction among implementation partners emerged as unintended sideeffects.

\section{Discussion}

The literature review, presented in this study, highlights different ways in which ISDM has been conceptualized and addressed with regard to particular disciplinary concerns. Four theoretical perspectives on ISDM were identified: system; structure; knowledge; and innovation. While each perspective regarded in isolation provides different overarching interpretations of ISDM, together they provide a more nuanced picture of ISDM and its potential value in different contexts.

Our analysis shows the circumstances under which the interplay between situational logics and implementers' stances engenders changes in the instantiation of IM, and thereby contributes to variations in implementation method instantiations. By theorizing about the emergence of the IM as constituted by the interplay between situational logics and implementers' stances it is possible to identify four IM instantiations which can be illustrated as a fourcategory classification:

A fragmented IM instantiation, involving a limited extension of the IM content by adding cognitive and production support, achieved by passive implementers with disconcerted concerns in a situation of compromise;

An aggregated IM instantiation, involving a horizontal expansion of the IM content by adding control and analysis support, achieved by evasive implementers with an ultimate concern in maintaining concordant inter-relationships in a situation of protection;

An integrated IM instantiation, involving a vertical expansion of IM content by adding cooperation and representation support, achieved by subversive implementers with an ultimate concern in exposing best practices, in a situation of opportunity; and

An infrastructural IM instantiation involving a connective extension of the IM content by adding organizational, coordination and production support, in a comprehensive platform of service and resources achieved by strategic implementers with an ultimate concern on proficiency and feasible performance achievements in situation of opportunity.

The taxonomy yields insights into IM instantiations and is intended to provide a classification of potential instantiations rather than a sequence and a list of all possible IM instantiations. With regard to this sequence, some tendencies are worth noting.

First, the first two IM instantiations denote situations of correction and protection, which in conjunction with passive or evasive agential stances contribute to reproduction of social context, i.e., morphostatis. The latter IM instantiations denote situations of opportunism, which in conjunction with subversive or strategic agential stances, contribute to a transformation of social context, i.e., morphogenesis.

Second, the first and the last IM instantiations indicate a productivity-oriented agency whose individualized concerns are placed within an organization's own action context. By contrast, the second and the third IM instantiations indicate a relationship-oriented agency whose main concerns is on preservation or transformation of collectively shared action concerns.

The conceptual framework applied in this study exemplifies how a morphogenetic approach might be used to provide a coherent view of variations in implementation methodology instantiations and their generation over time. It also adds more precision in explaining different IM instantiations and what contributes to these variations.

\section{Conclusion and further research}

This study has provided an alternative conceptualization of the implementation methodology instantiations, one informed by a morphogenetic approach underpinned by a critical realism perspective. The following research question is answered: How are implementation methodology instantiations generated over time?

We have identified four theoretically and empirically grounded implementation methodology instantiations which emerge from the interplay between situational logics and implementers' stances.

The intensive longitudinal case study focused on a single implementation methodology, i.e., ASAP, limiting the generalizability of our results beyond SAP's implementation methodology. Therefore, it is partial but has been developed by corroborating theoretical and empirical evidence. Nevertheless, this study makes a number of contributions.

First, the suggested framework helps IS researchers in identifying and explaining IM instantiations by taking into account the conditions under which these instantiations emerge over time.

Second, this study has found evidence for the usefulness of critical realism for developing substantive contributions in the IS field. Specifically, the morphogenetic approach has helped explain variations in IM instantiations over time. 
Third, understanding the conditions and the outcomes of IM instantiations might assist IS implementers in formulating design propositions, which might provide guidance for practitioners confronted by managing implementation methodologies.

Besides the theoretical contribution, the results from this study can also help practitioners to become aware of the features of IM instantiations. There is much research still to be carried out on this subject. For example, further work can study the interplay other situational logics and agent's stances which might generate alternative IM instantiations.

\section{References}

[1] M.S. Archer, Realist Social Theory: The Morphogenetic Approach, Cambridge University Press, Cambridge, 1995.

[2] M.S. Archer, Making our Way through the World, Cambridge University Press, Cambridge, 2007.

[3] M.S. Archer, Morphogenesis: Realism's explanatory framework, in Sociological realism, Taylor \& Francis, 2011.

[4] D.E. Avison and G. Fitzgerald, Information Systems Development: Methodologies, Techniques and Tools" 4th ed. McGraw-Hill, New York, 2006.

[5] P. Beynon-Davies, P. and M.D. Williams, "The diffusion of information systems development methods", Journal of Strategic Information Systems", (12), pp. 29-46 2003.

[6] B. Danermark, B. M. Ekström, L. Jakobsen and J. Karlsson, Explaining Society: Critical realism in the social sciences, London: Routledge, 2002).

[7] H.T. Davenport, Mission Critical: Realizing the promise of Enterprise Systems, Harvard Business School Press, 2000.

[8] P. Dobson, P. The philosophy of critical realism - an opportunity for information systems research, Information Systems Frontiers, July, 2011.

[9] P. Dobson, P. Jackson, and D. Gengatharen, "Explaining Broadband Adoption in Rural Australia: Modes of Reflexivity and the Morphogenetic Approach", MIS Quarterly, (37:3), pp.965-991, 2013.

[10] J. Esteves and V. Bohorquez, "An Updated ERP Systems Annotated Bibliography: 2001-2005", Communication of the Association for Information Systems, 9, pp. 386-446, 2007.

[11] R.G. Fichman and C.F. Kemerer, "The Assimilation of Software Process Innovations: An Organizational Learning Perspective", Management Science, (43:10), 1997.
[12] M. Huisman, and J. Iivari, "Deployment of systems development methodologies: Perceptual congruence between IS managers and systems developers", Information \& Management, (43), pp. 29-49, 2006.

[13] J. Iivari, and M. Huisman, "The Relationship between Organizational Culture and the Deployment of Systems Development Methodologies”, MIS Quarterly (31:1), 2007.

[14] S.T. March, and G.F. Smith, "Design and natural science research on information technology", Decisions Support Systems, (15), pp. 251-266, 1995.

[15] J. Mingers, "Real-izing Information Systems: Critical Realism as an Underpinning Philosophy for Information Systems", Information and Organization (14:2), 2004.

[16] J. Mingers, A. Mutch, and L. Willcocks, "Critical realism in information systems research", MIS Quarterly, (37:3), pp. 795-802, 2013.

[17] L.F. Motiwalla, and J. Thompson, Enterprise Systems for Management, New Jersey: Pearson Education, 2012.

[18] S. Newell, J.A. Swan, and R.D. Galliers, "A knowledgefocused perspective on the diffusion and adoption of complex information technologies: the BPR example", Information Systems Journal, (10:3), pp. 239-259, 2000.

[19] A.G. Nilsson, Enterprise Systems in a Service Science Context, in Information Systems Development: Asian Experiences, Boston: Springer, pp. 15-26, 2011.

[20] W. Orlikowski, (1992). "The duality of technology: rethinking the concept of the technology in organizations", Organization Science, (3), pp. 398-427, 1992.

[21] G. Fisher, and J. Ostwald, Knowledge Communication in Design Communities, in Barriers and Biases in ComputerMediated Knowledge Communication, Kluwer Academic Publisher, Netherlands, pp. 1-32, 2003.

[22] E.M. Roger,). Diffusion of Innovations, The Free Press, New York, 1995.

[23] A. Sayer, Realism and social science, London, 2000.

[24] D.P. Truex, and D. Avison, "Method Engineering: Reflections on the Past and Ways forward", AMCIS, 2003.

[25] V. Venkatesh, M.G. Morris, G.B. Davis, and F.D. Davis, "User Acceptance of Information Technology: Towards a Unified View”, MIS Quarterly, (27:3), pp. 425-478, 2003.

[26] P.J. Ågerfalk, and B. Fitzgerald, Exploring the concept of method rationale: a conceptual tool for method tailoring, Idea Group, Hershey, US 2006.

[27] J.M. Njihia, and Y. Merali, "The Broader Context for ICT4D Projects: A Morphogenetic Analysis”, MIS Quarterly, (37:3), pp. 881-905, (2013). 